\title{
Induced Short-Term Hearing Loss due to Stimulation of Age-Related Factors by Intermittent Hypoxia, High-Fat Diet, and Galactose Injection
}

\author{
Dong Jun Park ${ }^{1}$, Sunmok Ha ${ }^{2}$, Jin Sil Choi ${ }^{1}$, Su Hoon Lee ${ }^{1}$, Jeong-Eun Park ${ }^{3}$ and Young Joon \\ Seo ${ }^{1, *}$ \\ 1 Department of Otorhinolaryngology, Yonsei University Wonju College of Medicine, 20 Ilsan-ro, Wonju, \\ Gangwon-do 26426, South Korea. \\ 2 Department of Biomedical Laboratory Science, College of Health Sciences, Yonsei University, wonju, \\ Republic of Korea. \\ 3 Department of Otorhinolaryngology Head and Neck Surgery, Hallym University College of Medicine, \\ Dongtan Sacred Heart Hospital, Hwaseong, South Korea. \\ * Correspondence: okas2000@hanmail.net; Tel.: +82-33-741-0644 (Y.J.S.)
}

\begin{abstract}
Age-related hearing loss (ARHL) is the most common sensory disorder in the elderly. It is associated with aging and hair cell death due to oxidative stress-induced mitochondrial dysfunction. Although transgenic mice and long-term cultures for induction of aging have been used to study ARHL, there are presently no ARHL animal models stimulated by intermittent environmental change for aging. In this study, an ARHL animal model was established by inducing continuous oxidative stress to promote short-term aging of cells, determined based on the expression of the hearing loss-induced phenotype and aging related factors in the short term. The incidence of hearing loss was significantly different among the groups subjected to intermittent hypoxic environment, high-fat diet (HFD), and injection with D-galactose. Continuous oxidative stress and HFD were factors that accelerated cellular aging. Increase in UCP2 affected oxidative stress and mitochondrial dysfunction. CDH23, SLC26A4, KCNQ4, Myo7a, and Myo6, which are ARHL-related factors, were modified by oxidative stress in cells of the hearing organ. We found that intermittent hypoxic, HFD, and galactose injection accelerated cellular aging in the short term. Thus, we anticipate that the development of this hearing loss animal model, which reflects intermittent environmental changes, will benefit future research on ARHL.
\end{abstract}

Keywords: Mitochondria dysfunction; Reactive oxygen species; Hypoxic; D-galactose; High fat diet; aging; hearing loss

\section{Introduction}

Age-related hearing loss (ARHL), also called presbycusis, is an emerging complication in an ageing population worldwide. Because the gradual decrease of the hearing function with increasing age is often perceived as an inevitable part of the human aging process, the overall contribution of ARHL to hearing impairment and quality of life is underestimated. However, since the average life expectancy of the population is increasing, personal well-being has significant implications for hearing loss in general health and for extended life [1,2]. Various clinical reports have investigated ARHL. Fifteen percent of individuals between 50 and 59 years of age are bilaterally deaf, while $19 \%$ of individuals aged $40-69$ and $43.2 \%$ of those aged over 70 have the same condition in US National Health and Nutrition Examination Survey in 2015 [3]. Based on clinical results, the aged related patients will increase exponentially by a factor of 2 [2].

Most studies on ARHL have aimed at its prevention and treatment and require a long period of aging for preclinical evaluation. Factors and causes of aging have been studied using genetically engineered mice to uncover mechanisms of aging and to discover therapeutic drugs [4]. It was confirmed that oxidative stress caused by reactive oxygen species (ROS) is involved in cochlear cell 
death in transgenic mice that inhibited apoptosis-related bax. In fact, mitochondrial DNA damage caused by aging was observed to induce hearing cell death. Similarly, the expression of superoxide dismutase 1 (SOD1) or Cadherin 23 (CDH23) has been reported to be associated with aging. In addition, numerous reports suggest that abnormal potassium channels resulting in mass transfer errors cause cell death $[5,6]$. However, these animal models have certain limitations since they do not reflect the lifestyle of the animals [4,7]. Cumulative damage caused by the surrounding environment contributes to ARHL. Oxidative stress accelerates the aging of auditory cells, ultimately causing hearing loss [8]. Therefore, developing a preclinical animal model to study the effect of drugs over a short period by utilising a model in which oxidative stress is induced by simulating changes in environmental conditions is essential [9].

We previously conducted studies related to hearing loss due to hypoxia. Association between decreased oxygen saturation and hearing loss has been previously reported [10,11]. Therefore, in this study, we used a method based on the induction model of obstructive sleep apnoea syndrome (OSAS), wherein cell aging was promoted by temporarily blocking the supply of oxygen [12]. This model is designed such that the increase in ROS in the blood damages the auditory organs over a short period. We hypothesised that ARHL could be detected by observing changes in the aging phenotype caused by the intermittent hypoxic environment.

Mitochondrial damage has been attributed for the death of auditory blast cells due to aging $[13,14]$; therefore, based on literature identifying relevant factors, animal models were designed according to their lifestyle $[15,16]$. It has been reported that mitochondrial dysfunction can also be induced by modifying the diet [17]. High-fat diet resulted in increased intracellular lipid content, diabetes-induced symptoms, and impairment of mitochondrial function due to oxidative stress. Caloric regulation associated with deafness has been reported to suppress cell aging through the inhibition of Foxo3 and Sirt1 expression, as well as apoptosis-related proteins [18]. In addition, clinical studies have reported that over $50 \%$ of patients with diabetes have hearing loss and if diabetes persisted for more than 5 years, the hearing loss rate would double [19]. D-galactose (D-gal) injection animal models, established by administering successive subcutaneous D-gal injections to animals for approximately 6 or 8 weeks, have been frequently used in aging studies. Accelerated aging factors in rodents induced by D-gal was reported by Guo et al. [20]. In addition, animal models using the galactose injection have been proven to accelerate aging of the brain, kidney, liver, and blood cells [21-23].

Therefore, we developed an ARHL animal model by stimulating the aging factors of mice exposed to intermittent oxidative stress in the short term by changing various aspects of lifestyle of the mice to induce the death of auditory hair cells in the Organ of Corti (OC) and hearing-related factors caused by aging. This study provides a realistic animal model that can be used to accelerate the development of therapeutic strategies for senile hearing loss in the future.

\section{Materials and Methods}

\subsection{Experimental groups}

A total of 72 mice were divided into eight groups (Figure 1), some of which were exposed to intermittent oxidative stress while others were used as controls. The different groups were as follows: Group 1 (G1), Normoxic, Normal Feed (NF); Group 2 (G2) Normoxic, NF, Galactose injection GI; Group 3 (G3), Normoxic, High fat diet (HFD); Group 4 (G4), Normoxic, HFD, GI; Group 5 (G5), Hypoxic, NF; Group 6 (G6), Hypoxic, NF, GI; Group 7 (G7), Hypoxic, HFD; and Group 8 (G8), Hypoxic, HFD, GI. Additionally, young mice (male, 4 weeks, $n=9$ ) were used as controls. Mice body weight was monitored throughout the experiment as an indicator of health. 


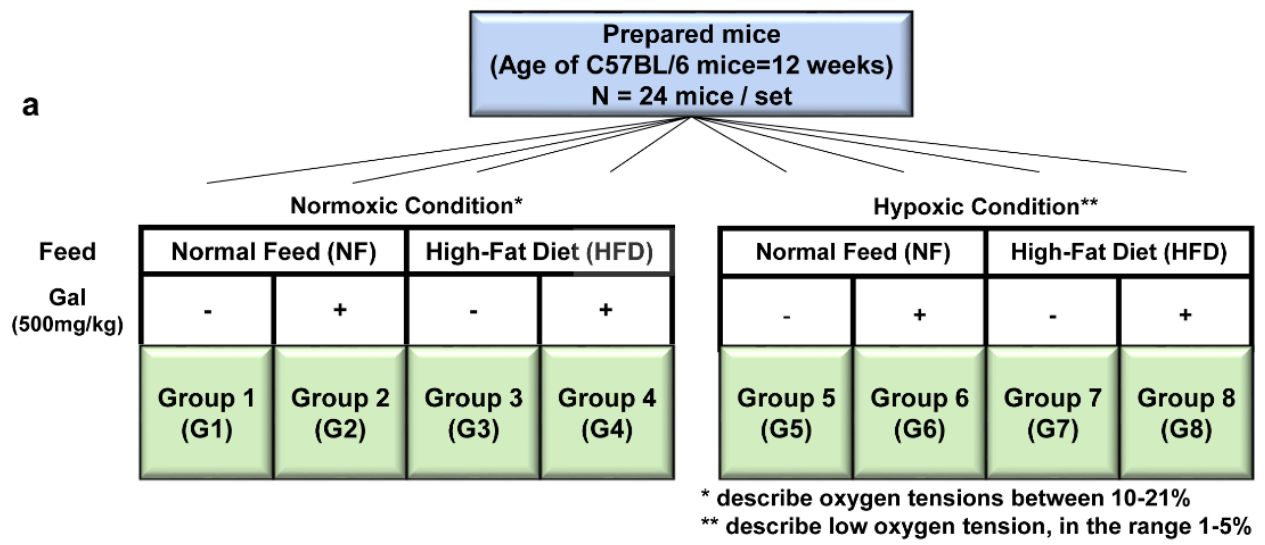

b

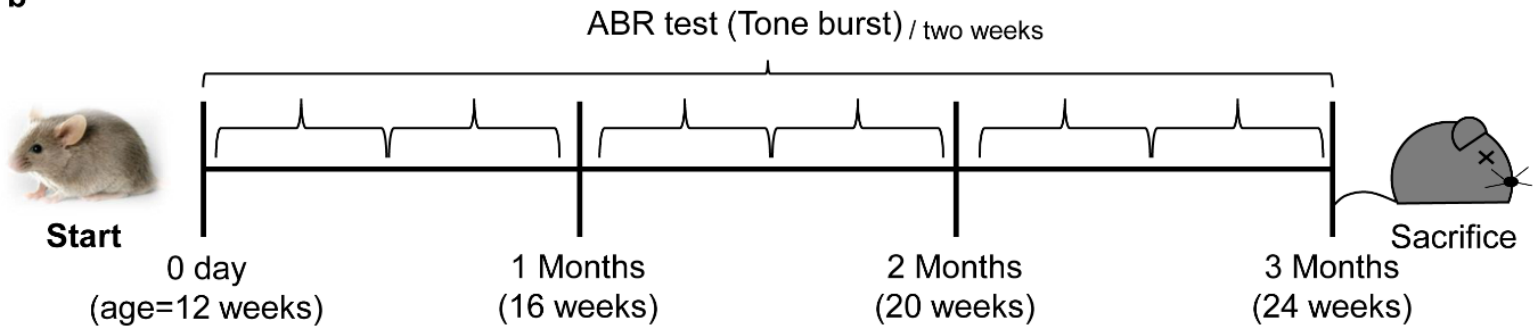

Figure 1. Overall design, including the mouse housing conditions and ABR test. (a) Classification of groups based on hypoxic condition ( $\leq 5 \%)$, HFD, and galactose injection $(500 \mathrm{mg} / \mathrm{kg})$. Mice $(\mathrm{n}=72)$ were divided into eight groups in this study. G1 (Normoxic, NF), G2 (Normoxic, NF, GI), G3 (Normoxic, HFD), G4 (Normoxic, HFD, GI), G5 (Hypoxic, NF), G6 (Hypoxic, NF, GI), G7 (Hypoxic, HFD), and G8 (Hypoxic, HFD, GI). (b) The ABR test for all mice was performed once every two weeks. Starting at 12 weeks of age, all mice were sacrificed by Cervical dislocation after 3 months when hearing loss was measured.

\subsection{Animal procedures}

Male C57BL/6 mice (12 weeks old) were used in this study. All animal procedures were approved by the institutional animal care and use committee in the animal laboratory of Yonsei University in Wonju College of Medicine (Permit Number: YWC-181001-2). All animals were maintained at room temperature with a 12-h light/dark cycle under different oxygen conditions, such as normoxic and intermittent hypoxic conditions. They were classified according to three conditions for each group. G1 to 4 were kept in normoxia with an oxygen concentration of $20 \%$, while groups 5 to 8 were kept in a hypoxia chamber with an oxygen concentration of $5 \%$ for $12 \mathrm{~h} /$ day. In addition, the mice were divided into normal feed (NF) (NIH-41, autoclaved, Zeigler Bros Inc., PA, USA) [24] and high-fat diet (HFD) groups [17]. The HFD was prepared as previously reported [16]. All ingredients and supporting data are listed in Table S1. G3, 4, 7, and 8 were fed an HFD with a fat content of 32\% (Table S1), including vitamins (Table S4), to generate oxidative stress in the body, and groups 1, 2, 5, and 6 were fed NF (Table S2). The body metabolism changes during HFD to promote the aging of cells (Table S3).

In addition, G2, 4, 6, and 8 were designed to evaluate the effect of promoting aging through galactose injection $(500 \mathrm{mg} / \mathrm{kg})$ to cause chronic oxidative stress and mitochondrial dysfunction. D-galactose (G0750, Sigma-Aldrich, USA) was dissolved in $0.9 \%$ saline solution and subcutaneously injected to induce aging in several groups $(500 \mathrm{mg} / \mathrm{kg}$ ) while other groups were injected with an equal volume of vehicle ( $0.9 \%$ saline).

Body weight was measured weekly to monitor changes. Furthermore, the threshold of hearing was evaluated by measuring auditory brainstem response (ABR) every two weeks. In order to check the oxidative stress in the body, serum samples were collected every month. 


\subsection{Hypoxia chamber design}

The hypoxia chamber was designed to inflict chronic oxidative stress in the mice (Figure 2). This chamber was made of acrylic sheets and consisted a fan that automatically injected fresh air and nitrogen into the upper part of the chamber and dispersed the air (Figure S1). The hypoxia chamber $(340 \times 240 \times 60 \mathrm{~mm}$, internal volume $4.9 \mathrm{~L})$ was designed to control oxygen concentration via nitrogen injection with LCI (Live Cell Instrument Co., Seoul, Korea) in accordance with previous literature $[12,25]$. This chamber automatically controlled the oxygen concentration at about $20 \%$ and $5 \%$ using nitrogen at a 12-h time split. Nitrogen was automatically injected every $2 \mathrm{~min}$ during the $12 \mathrm{~h}$ period to decrease oxygen concentration to $5 \%$. After $12 \mathrm{~h}$, fresh air was injected to allow sufficient oxygen for $12 \mathrm{~h}$. In total, a 24-h reaction was performed in each cycle and the concentration of oxygen was recorded every second per day (Figure $2 b, c)$. All the used gas was discharged out of the building via tubes and oxygen indicator was used to check the amount of oxygen in the hypoxia chamber. Inside the chamber, walls were used to divide the NF and HFD groups to distinguish the type of feeding (Figure 2d). The cages were cleaned once a week and feed, water, and defecation by mice were removed.
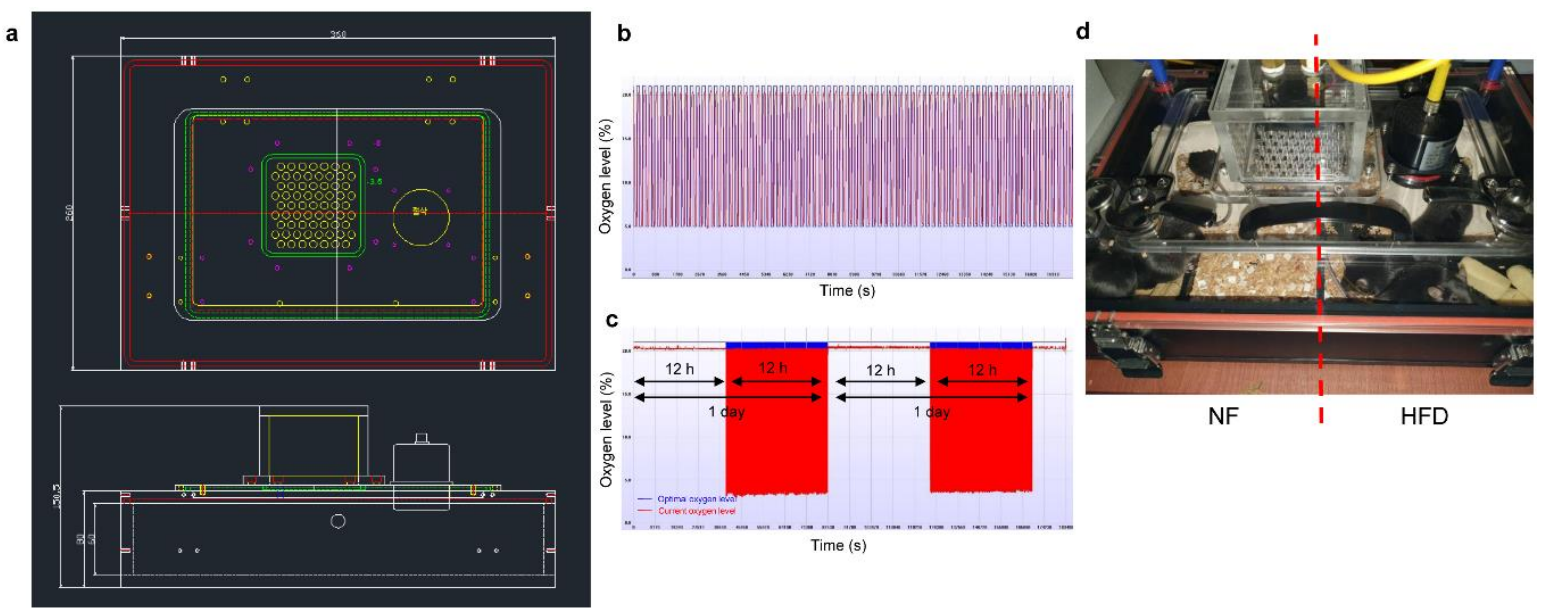

Figure 2. Hypoxia chamber design for oxidative stress. (a) Blueprint of the hypoxia chamber for mice. (b) Measurement of automatic change in oxygen levels in the hypoxia chamber from $20 \%$ to $5 \%$. The blue line represents the amount of oxygen set by the machine, and the red line represents the actual amount of oxygen. Nitrogen was automatically provided into the chamber to alter the oxygen levels. (c) Daily analysis for the oxygen level in the chamber. Oxygen was cycled through the chamber to obtain the hypoxic condition $(\leq 5 \%)$ for $12 \mathrm{~h}$ a day and the normoxic condition $(20 \%)$ for $12 \mathrm{~h}$ a day. (d) The mice were physically divided by the middle wall in the hypoxia chamber. Mice on the left side of the chamber were administered NF, while those on the right side were fed a HFD.

\subsection{Auditory Brainstem Response (ABR)}

All mice were anesthetised with $100 \mathrm{mg} / \mathrm{kg}$ ketamine (Yuhan-Kimberly, Seoul, Korea) and 10 $\mathrm{mg} / \mathrm{kg}$ xylazine hydrochloride (Rompun, Bayer, Ansan, Korea) by intraperitoneal injection before $A B R$ recording. Two ABR measurement methods were used in this study. The clicking sound was measured by reducing the $10 \mathrm{~dB}$ range from $90 \mathrm{~dB}$ to $10 \mathrm{~dB}$ on $8,000 \mathrm{~Hz}$ for accurate measurement. It was also measured by reducing $5 \mathrm{~dB}$ from $80 \mathrm{~dB}$ to $20 \mathrm{~dB}$ for each frequency $(4,000,8,000,16,000$, 24,000 , and $32,000 \mathrm{~Hz}$ ) to observe age-related hearing loss.

Mice were tested in a sound attenuating chamber with a built-in Faraday cage and an isothermal pad was used to maintain the body temperature. The TDT RZ6/BioSigRZ system (Tucker Davis Technologies, Alachua, FL, USA) was used for stimulus generation, data management, and ABR collection [26]. Subdermal electrodes were placed in each mouse for data collection. The reference electrode, which was on the same side as the stimulus, was placed axial to the pinnae, while the 
ground electrode was placed in the ipsilateral ear. Meanwhile, the active electrodes were placed in the vertex. The ABR test was conducted every two weeks for the stability of the experimental group.

2.1 Superoxide dismutase (SOD) activity test

SOD was rapidly measured using blood collected from the retro-orbital plexus of mice in each group. The collected blood was allowed to clot in an anticoagulant tube for $30 \mathrm{~min}$ at $25^{\circ} \mathrm{C}$ (Room temperature, RT). Purified serums were obtained after the blood was centrifuged at 2,000 $\mathrm{g}$ for 15 min at $4^{\circ} \mathrm{C}$. All samples were stored at $-80^{\circ} \mathrm{C}$ in stock for a month and working samples were diluted 1:5 with sample buffer before assaying for SOD activity. The Superoxide Dismutase Assay kit (No. 706002, Cayman Che., MI, USA) uses a tetrazolium salt for detection of superoxide radicals generated by xanthine oxidase and hypoxanthine [27]. For each sample, SOD activity was calculated using the equation obtained from the linear regression of the standard curve replacing the linearized rate. One unit was defined as the amount of enzyme required to represent $50 \%$ displacement of the super oxide radical. All SOD activities were measured according to the proposed method.

\subsection{Histological analysis}

All mice were sacrificed by Cervical dislocation and both cochleae were dissected. The cochleae were perfused with a fixative containing $4 \%$ paraformaldehyde in phosphate-buffered saline ( $\mathrm{pH} 7.4)$ at RT. The apical portion of the bony cochlea was gently opened to allow the fixative to perfuse through the tissues. Cochleae were decalcified by immersion in Calci-Clear Rapid (National Diagnostics, Atlanta, GA) for $24 \mathrm{~h}$, then embedded in a compound at optimal cutting temperature (Leica Microsystems, Bensheim, Germany), and cut into 2- to $5-\mu$ m-thick sections in a LEICA RM2145 (Leica Biosystems, Wetzlar, German), which were subjected to standard hematoxylin and eosin (H\&E) staining (1-3 min of incubation in hematoxylin and staining with eosin for 30-60 s).

\subsection{Immunostaining}

Cochlea sections were prepared on 5 - $\mu$ m-thick gelatin-coated slides, then fixed in $4 \%$ paraformaldehyde for $15 \mathrm{~min}$ and allowed to dry at RT. The specimens were incubated in $5 \%$ normal goat serum for $1 \mathrm{~h}$ at $\mathrm{RT}$ to prevent non-specific labelling and were then incubated with the primary antibody, Myo7a (1:200,ab3481, Abcam, UK), for $1 \mathrm{~h}$ at $4^{\circ} \mathrm{C}[28,29]$, followed by incubation with a secondary antibody, goat anti-rabbit IgG H\&L (Alexa FluorR 488) ((1:1000, ab150077, Abcam, UK) for $1 \mathrm{~h}$ at RT after washing the specimens three times for $5 \mathrm{~min}$ with PBS. Lastly, the samples were immobilised with mounting solution containing DAPI after the specimens were washed three times for 5 min with PBS. All samples were observed by confocal microscopy (Carl Zeiss Microscopy $\mathrm{GmbH}$, Jena, Germany) and images were analysed using the software ZEN lite ver. 2.3.

\subsection{Scanning electron microscope (SEM)}

The cochlea was isolated to observe the morphology of the hair cell. The cochlea was extracted from the auditory organ and fixed in $2.5 \%$ glutaraldehyde for $2 \mathrm{~h}$ at $4^{\circ} \mathrm{C}$. The specimens were then fixed in $1 \%$ osmium tetroxide $\left(\mathrm{OsO}_{4}\right)$ after being washed twice with $0.1 \mathrm{M}$ cacodylate buffer. The dehydration steps were performed using 50, 70, 80, 90. and 100\% ethanol. Then, the samples were made to react with 3-methylbutyl acetate (Isoamyl acetate, Hanawa, Japan) for $15 \mathrm{~min}$ at RT. The samples were dried using Hexamethyl disilazane (cat. 440191, Sigma-Aldrich, USA) for 15 min at RT. All samples were air dried with the lid on the filter paper overnight. Gold coatings were performed to observe the morphology of the cochlea using a tabletop microscope (TM-1000, Hitachi Ltd., Tokyo, Japan) [30].

\subsection{Real time-PCR}

Real time-PCR was performed to determine the induction of aging and hearing loss by analysing the expression of various genes in the liver, kidney, and cochlea. Total RNA was extracted using TRIzol (Thermo Fisher Scientific). To prepare mRNA samples, $2 \mu \mathrm{L}$ of mRNA and $8 \mu \mathrm{L}$ of reverse transcriptase reagents, which comprised $1 \mu \mathrm{L}$ of $10 \times$ enzyme mix, $2 \mu \mathrm{L}$ of $5 \times$ enzyme reaction 
buffer, and $5 \mu \mathrm{L}$ of nuclease-free water, were used to prepare a $10 \mu \mathrm{L}$ mixture according to the manufacturer's protocol. CDNA was diluted 1:10 with $90 \mu \mathrm{L}$ nuclease-free water for microRNA realtime PCR. RT-PCR was performed using the Applied Biosystems sequence detection system 7900 (Thermo Fisher Sci. MA, U.S.A.). Samples were subjected to reverse transcription using the SYBR Select master mix (Applied Biosystems, CA, USA) following the manufacturer's protocol.

The sequences of the primers are as follows (Table S6): Apo-lipoprotein E (ApoE), forward: 5'GGT TCG AGC CAA TAG TGG AA - 3' and reverse: 5'- ATG GAT GTT GCA GGA CA -3'; Cadherin23 (CDH23), forward: 5'- ATG GAG AGC CCT CTG GAA AT -3', and reverse: 5'- ACC CAC AAA GGC TGT ACT GG -3'; Eosinophil-derived neurotoxin 1 (EDN1), forward: 5'- ACA CCG TCC TCT TCG TTT TG -3', and reverse: 5'- GAG TC CTT GGA AAG TCA CG -3'; Potassium Voltage-Gated Channel Subfamily Q Member 4 (KCNQ4), forward: 5'- TGT TGG GAT CCG TGG TCT AT -3', and reverse: 5'- GAGTTG GCA TCC TTC TCA GC -3'; Myosin VIIA (Myo7a), forward: 5'- GAC AAC TCT AGC CGC TTT GG -3', and reverse: 5'- GAC ACG TGA CTT CTC CAG CA -3'; Myosin VI (Myo6), forward: 5'- AGA CCA CTT CCG GCT CAC TA - $3^{\prime}$, and reverse: 5'- TGG GTT GTC TCG TAG CAC AC -3'; Uncoupling protein 2 (UCP2), forward: 5'- CTC AAA GCA GCC TCC AGA AC -3', and reverse: 5'- ACA TCT GTG GCC TTG AAA CC -3'; Solute Carrier Family 26 Member 4 (SLC26A4), forward: 5'- TCA TTG CCT TTG GGA TAA GC - $3^{\prime}$ and reverse: 5'- GGC AAC CAT CAC AAT CAC AG -3'; $18 S$ rRNA, forward: 5'- CAT TCG AAC GTC TGC CCT AT - 3', and reverse: 5'- GTT TCT CAG GCT CCC TCT CC $-3^{\prime}$. The total $10 \mu \mathrm{L}$ sample for real-time PCR contained $1 \mu \mathrm{L}$ cDNA, $5 \mu \mathrm{L}$ of premix, 1 $\mu \mathrm{L}$ of 10 pmol forward and reverse primers, and $3 \mu \mathrm{L}$ of nuclease-free water. The PCR conditions comprised pre-amplification at $95^{\circ} \mathrm{C}$ for $3 \mathrm{~min}, 40$ cycles of $95^{\circ} \mathrm{C}$ for $10 \mathrm{~s}, 60^{\circ} \mathrm{C}$ for $60 \mathrm{~s}$ and melting curve analysis. All processes were performed in duplicate. Normalisation of mRNA expression level was calculated using $18 S$ rRNA.

\subsection{Statistical analysis}

Statistical analysis was performed using SPSS statistical package version 21.0 (SPSS Inc., USA). Descriptive results of continuous variables are expressed as the mean \pm standard deviation (SD) for normally distributed variables. Means were compared by 2-way analysis of variance. The sensitivity and specificity of the statistically significant mRNAs were analysed by Mann-Whitney $U$ test with GraphPad PRISM version 5.0 (GraphPad Inc., La Jolla, CA, USA). P-values less than 0.05 were considered to be statistically significant.

\section{Results}

\subsection{Phenotypic analysis of the different groups for aging}

The expected phenotype and hearing loss were observed at approximately 12 weeks. The mice were compared in terms of the various oxidative stress conditions such as HFD, galactose injection (GI), and hypoxia. The physical characteristics of mice in each group were also confirmed. In the group under oxidative stress, it was confirmed that the shine of the hair had disappeared, and the hair had a slight grey colour (Figure S2). The body weight of mice was significantly increased in the normoxic (Figure 3a) and hypoxic HFD groups (Figure 3b) compared to the control group at 3 months. First, in terms of the different feeding conditions, the control group showed a minor change in body weight of less than $3 \mathrm{~g}$, but the body weight in the HFD group had increased by approximately $20 \mathrm{~g}$ at 3 months from baseline at 0 months (Figure 3a). Under hypoxia, the control group showed a change in body weight within $4 \mathrm{~g}$, and the HFD group showed an increase by over $20 \mathrm{~g}$ at 3 months from baseline at 0 months (Figure $3 b$ ). In the group injected with galactose, an 8 to $10 \mathrm{~g}$ change in body weight under normoxic conditions was observed; however, under hypoxic conditions, a 3 to $6 \mathrm{~g}$ change in body weight was observed. Since we cannot determine aging based on changes in body weight alone, skin tissue from each group was collected to observe the changes in skin phenotype with aging. Interestingly, while an increase in body weight according to dietary conditions was observed, a change in skin thickness was also observed. The thicknesses of the dermis skin layer and fat layer were measured microscopically, and HFD was confirmed to show the highest effect on the 
fat layer [31-33]. The fat layer of mice in the HFD groups was the thickest (Table S5). In addition, hypoxia was confirmed to be the condition that most affected the dermis. An increase in thickness of about $100 \mu \mathrm{m}$ was observed under hypoxic conditions, and many deep wrinkles were observed on the skin surface in the GI group.

a
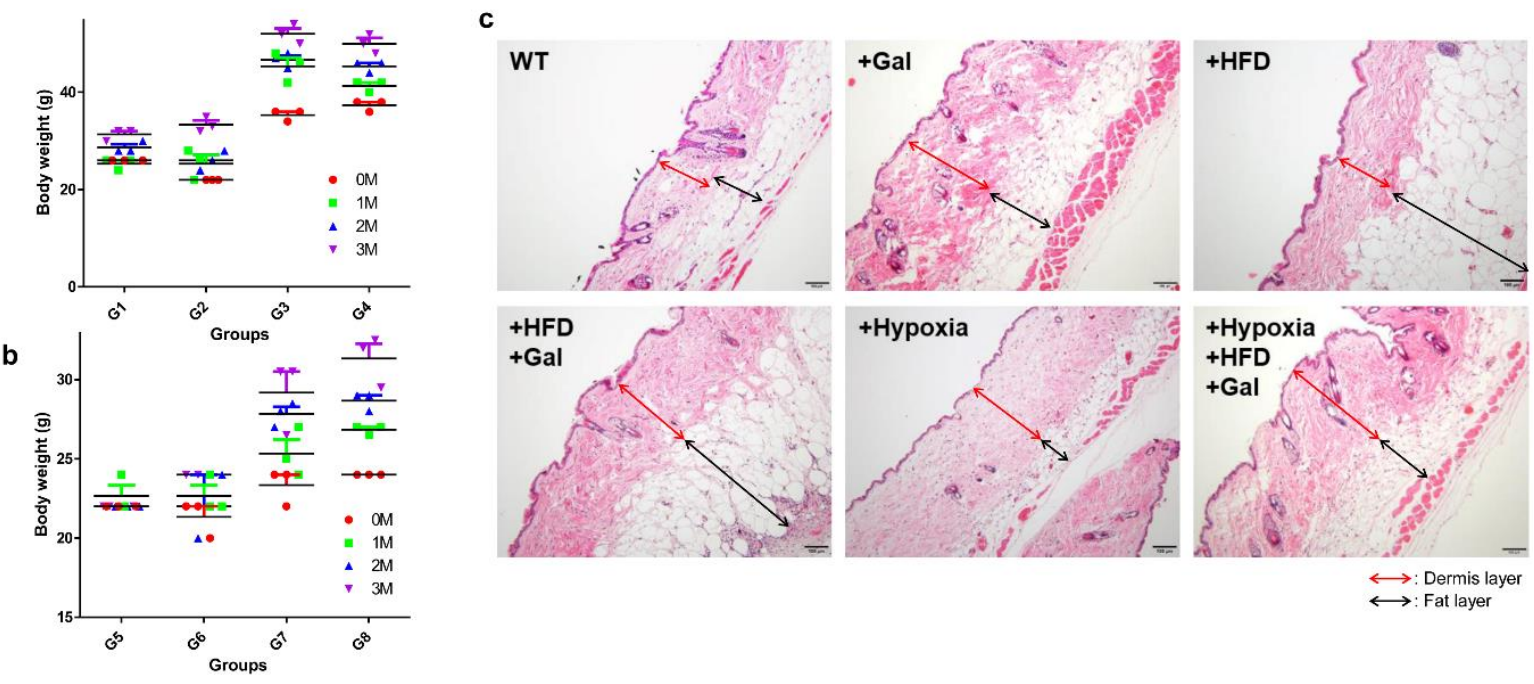

Figure 3. Phenotypic analysis of aging mice under oxidative conditions. (a) Measured body weight of the normoxic groups. (b) Measured body weight of the hypoxic groups. HFD also increased body weight regardless of the hypoxic environment. (c) The thickness of the dermis skin layer and the thickness of the fat layer were different. Interestingly, the fat layer was significantly thickened in the HFD groups, but it did not increase in size under hypoxic conditions although body weight increased. Rather, the dermis layer became thicker and a lot of wrinkles were observed on the skin surface.

\subsection{Oxidative stress in serum}

Oxidative stress has been demonstrated to be the most important factor that causes aging $[10,11]$. Therefore, increase in the ROS and SOD in serum could be a major reason for induction of aging. In this study, we collected serum from mice in each group throughout the month. The SOD was measured to indicate the amount of oxidative stress in each group (Figure 4). Interestingly, oxidative stress was increased in the hypoxic group after 2 months. Normoxic was associated with a tendency of decreased oxidative stress after 3 months (Table 1).

Table 1. Monthly measurement of SOD activity in serum.

\begin{tabular}{ccccc}
\hline Groups & 0 month & 1 month & 2 months & 3 months \\
\hline 1 & $0.6325 \pm 0.000$ & $0.5873 \pm 0.005$ & $0.5667 \pm 0.000$ & $0.5311 \pm 0.019$ \\
2 & $0.5955 \pm 0.017$ & $0.5463 \pm 0.005$ & $0.5216 \pm 0.005$ & $0.5148 \pm 0.028$ \\
3 & $0.5586 \pm 0.011$ & $0.5463 \pm 0.005$ & $0.5298 \pm 0.005$ & $0.4901 \pm 0.011$ \\
4 & $0.5873 \pm 0.005$ & $0.5298 \pm 0.005$ & $0.5011 \pm 0.011$ & $0.5572 \pm 0.050$ \\
5 & $0.5873 \pm 0.029$ & $0.5463 \pm 0.005$ & $0.5463 \pm 0.005$ & $0.6215 \pm 0.048$ \\
6 & $0.5914 \pm 0.000$ & $0.5627 \pm 0.005$ & $0.5463 \pm 0.005$ & $0.5695 \pm 0.059$ \\
7 & $0.5996 \pm 0.000$ & $0.5463 \pm 0.005$ & $0.5914 \pm 0.000$ & $0.6435 \pm 0.055$ \\
8 & $0.5750 \pm 0.011$ & $0.5463 \pm 0.005$ & $0.5380 \pm 0.005$ & $0.5955 \pm 0.022$ \\
\hline
\end{tabular}

First, the hypoxic control returned a SOD value of $0.6215 \pm 0.048$, while the SOD value for the normoxic control value was $0.5311 \pm 0.019$. The SOD values for the GI hypoxic and normoxic groups were observed to be $0.5695 \pm 0.059$ and $0.5148 \pm 0.028$, respectively. The SOD values for the hypoxic and normoxic groups under HFD were observed to be $0.6435 \pm 0.055$ and $0.4901 \pm 0.011$, respectively. Finally, when the hypoxic and normoxic groups were exposed to both HFD and GI, the SOD values 
were $0.5955 \pm 0.022$ and $0.5572 \pm 0.050$, respectively. The 0.04 difference in the SOD value is a significant error value, and can be regarded as a similar pattern. In other words, it was confirmed that the amount of SOD in the body increased with exposure to oxidative stress for a longer duration, and the difference in SOD values between hypoxic and normoxic groups was about 0.05 to 0.1534. Thus, it was confirmed that HFD and GI did not significantly influence the amount of oxidative stress.

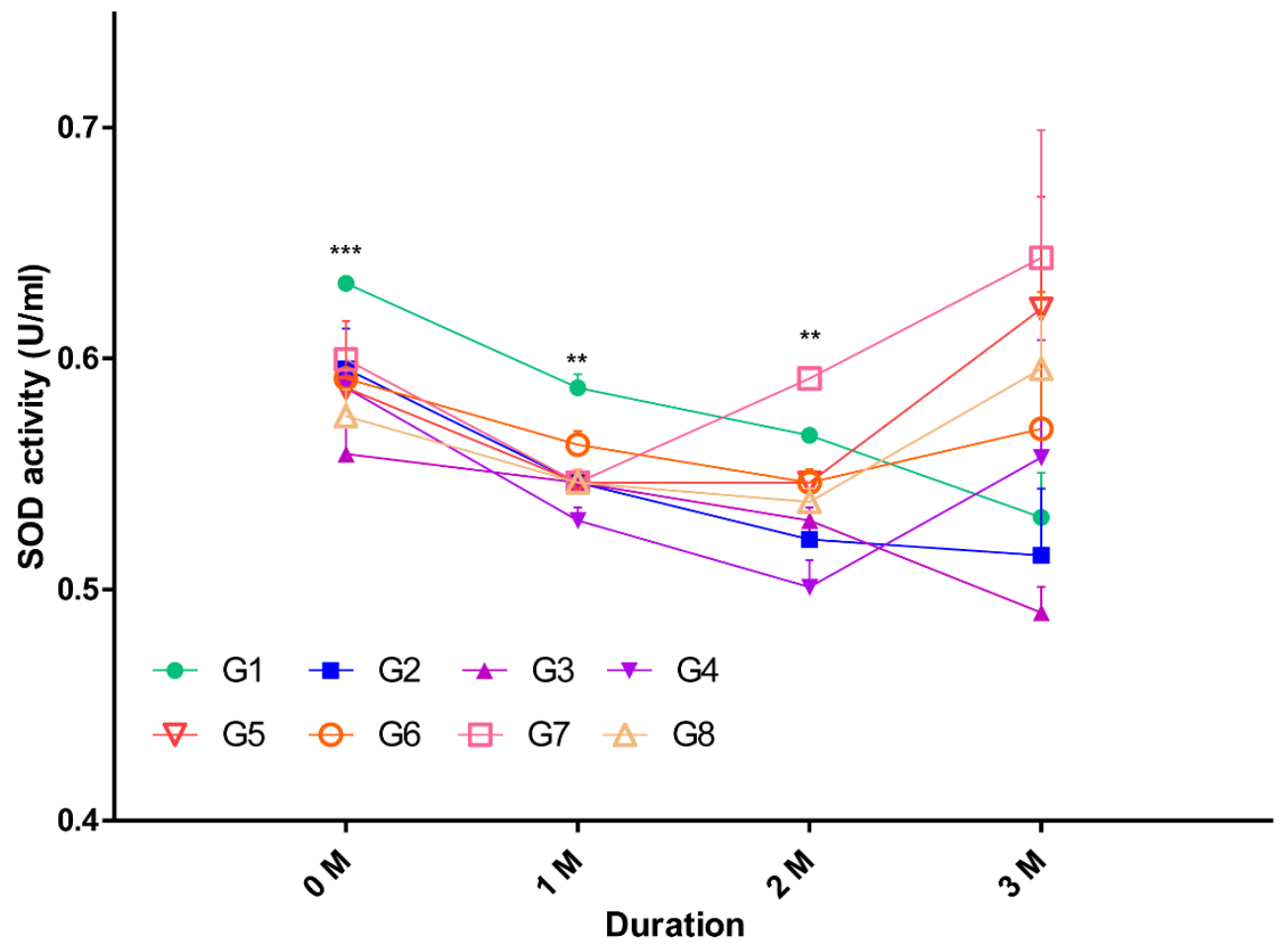

Figure 4. Monthly analysis of SOD activity in the serum from all groups. Serum was collected from mice in each group, and the amount of SOD in serum was measured. Significant values are shown for each month with ${ }^{*} \mathrm{p}<0.05,{ }^{* *} \mathrm{p}<0.005,{ }^{* * *} \mathrm{p}<0.0005$.

\subsection{Comparison of the hearing threshold for each period}

Hearing loss due to aging occurs at the highest to the lowest frequencies [4,34]. The hearing thresholds in the mouse groups were measured in the frequency range of $4 \mathrm{kHz}$ to $32 \mathrm{kHz}$ by toneburst $A B R$ (Figure 5). In the control group, the alteration in the hearing threshold was minimal during the 3 months (Figure 5a).

There was a significant decrease in the hearing threshold from $25 \mathrm{~dB}$ to $58 \mathrm{~dB}$ at $8 \mathrm{kHz}$ and 35 $\mathrm{dB}$ to $56 \mathrm{~dB}$ at $16 \mathrm{kHz}$ in the hypoxic group (Figure 5e). Conversely, a comparison of the results for the GI and HFD groups revealed that there was no observed decrease in the hearing threshold at all frequencies (Figure 5c, d). Next, three groups, HFD and GI (Figure 5f), hypoxia and GI (Figure 5g), and hypoxia and HFD (Figure 5h), were compared to confirm the dual effect. The hearing threshold was confirmed to have significantly reduced, from $34 \mathrm{~dB}$ to $67 \mathrm{~dB}$ in $8 \mathrm{kHz}$ and $38 \mathrm{~dB}$ to $60 \mathrm{~dB}$ in 16 $\mathrm{kHz}$, in the hypoxic condition. Finally, we evaluated the results for mice under all three conditions (hypoxia, HFD, and GI) to reduce hearing by ABR and designated them as the positive result. The reason for this is that the three conditions produced a significant effect and the value of hearing threshold significantly decreased after 3 months from $35 \mathrm{~dB}$ to $67 \mathrm{~dB}$ in $8 \mathrm{kHz}$ and $31 \mathrm{~dB}$ to $70 \mathrm{~dB}$ in $16 \mathrm{kHz}$ (Figure 5b). The hypoxic condition was observed to show the most effect, while the HFD and GI conditions showed the least effect, on hearing loss. 
a

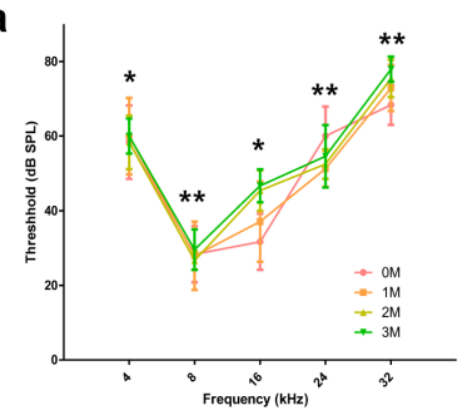

C

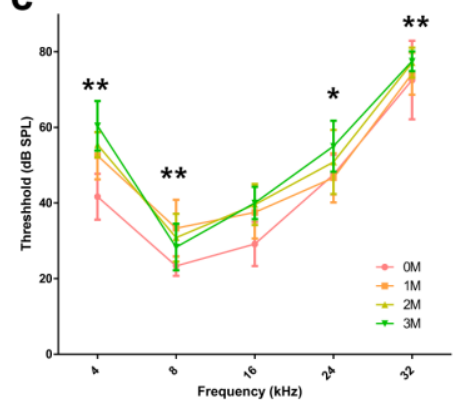

f

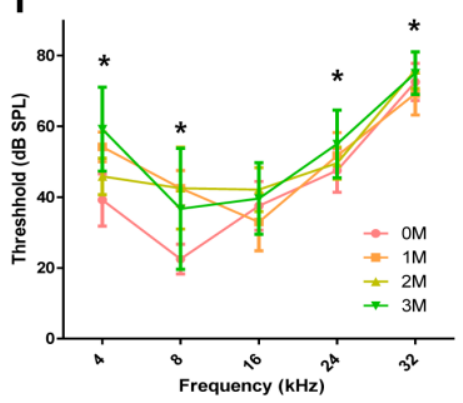

b

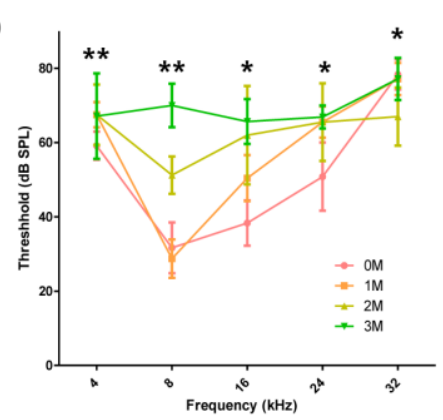

d

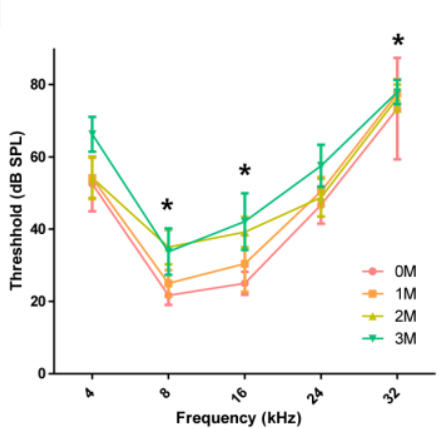

g

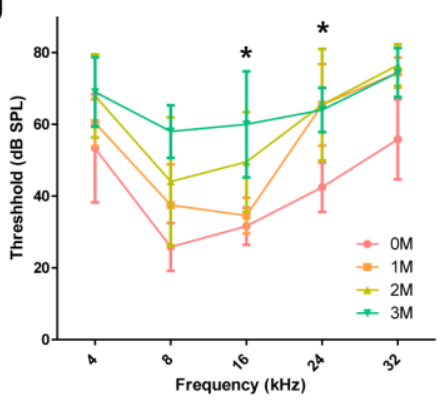

e

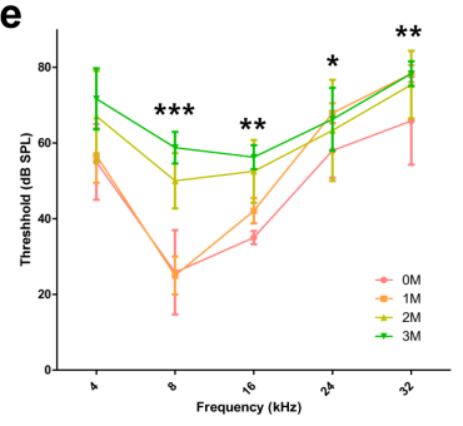

h

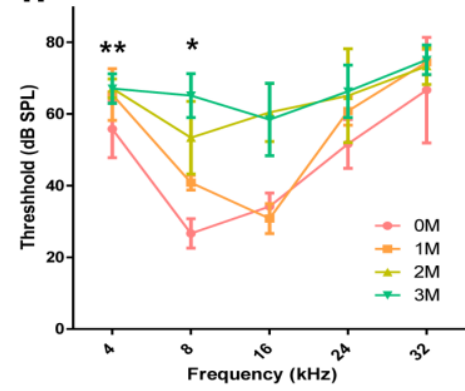

Figure 5. Comparison of the hearing threshold among all groups at various frequencies for each period using the ABR test. The threshold of hearing was found to be greatly decreased under the hypoxic condition. Galactose did not affect hearing loss alone, but led to hearing loss in combination with HFD and hypoxic condition. (a) Control group (G1), (b) Hypoxia, HFD, Galactose injection (GI) group (G8), (c) GI group (G2), (d) HFD group (G3), (e) Hypoxia group (G5), (f) HFD, GI group (G4), (g) Hypoxia, GI group (G6), and (h) Hypoxia, HFD group (G7). Significant values are shown with *p $<0.05,{ }^{* *} \mathrm{p}<0.005,{ }^{* * *} \mathrm{p}<0.0005$.

\subsection{Histological observations of hair cells}

We next evaluated the survival ratios of auditory hair cells under different conditions by histological analysis. The survival rate was evaluated by major protein, Myo7a, present in auditory hair cells. In 4-week-old mice, three outer hair cells (OHC) and one inner hair cell (IHC) were clearly observed (Figure 6a). In addition, no damage to hair cells was observed without oxidative stress after 3 months (Figure $6 \mathrm{~b}$ ). The results of the histological analysis showed that there was little damage under GI (Figure 6c), moderate damage under HFD (Fig. 6d), and severe damage under hypoxic conditions (Figure 6e). In addition, the analysis revealed that an OHC was on the brink of cell death in the dual and triple reactions (Figure 6f-h). For quantitative evaluation, we evaluated the survival rate of hair cells based on the histological images from each group (Figure 6i). The survival rate was over $80 \%$ in the single response and $50 \%$ in the dual response, but it was less than $20 \%$ when the hypoxic reaction was included. In other words, oxidative stress caused by hypoxia shows damage to hair cells and causes hearing loss. 

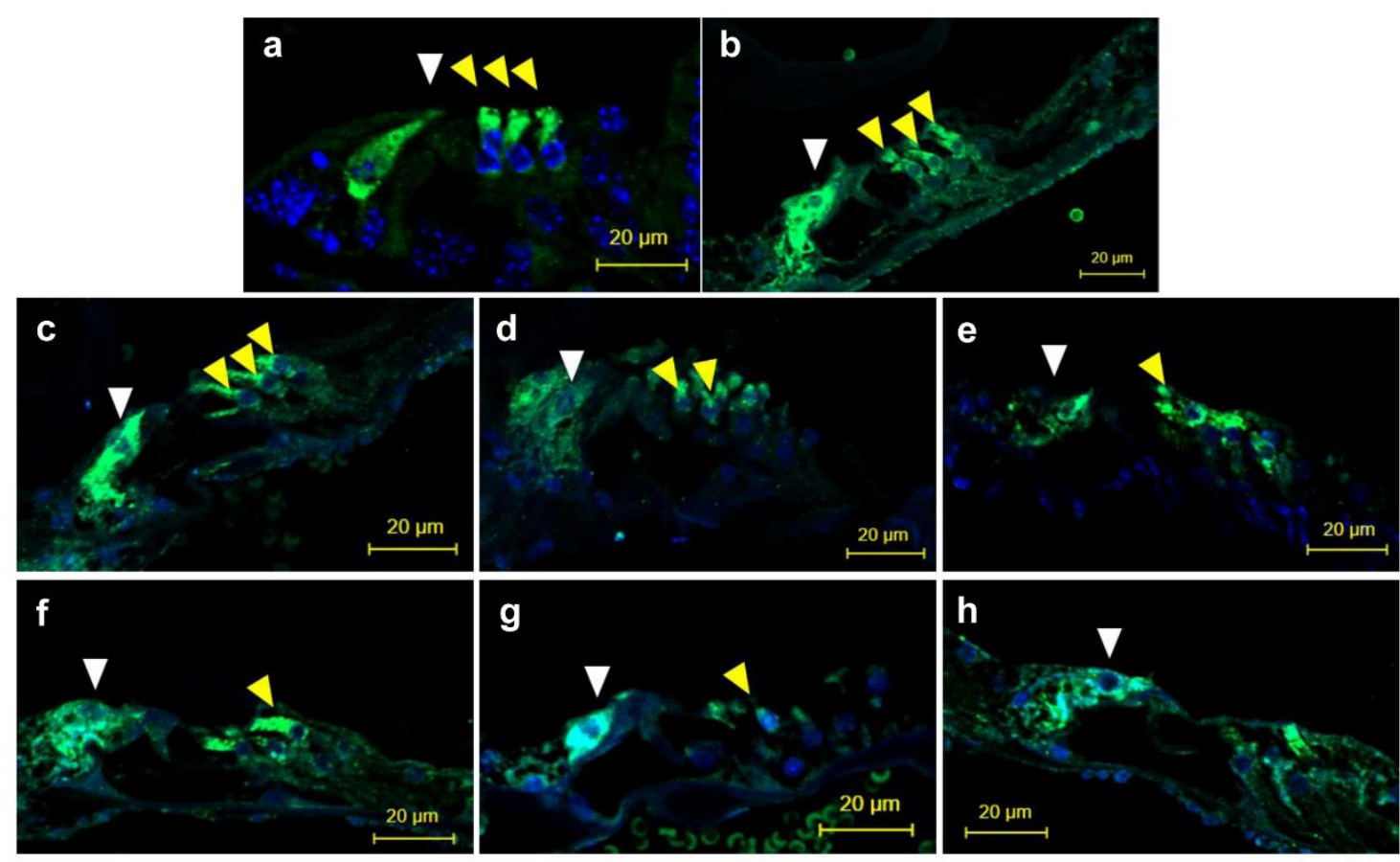

i

$\otimes \mathrm{IHC} \otimes \mathrm{OHC}$

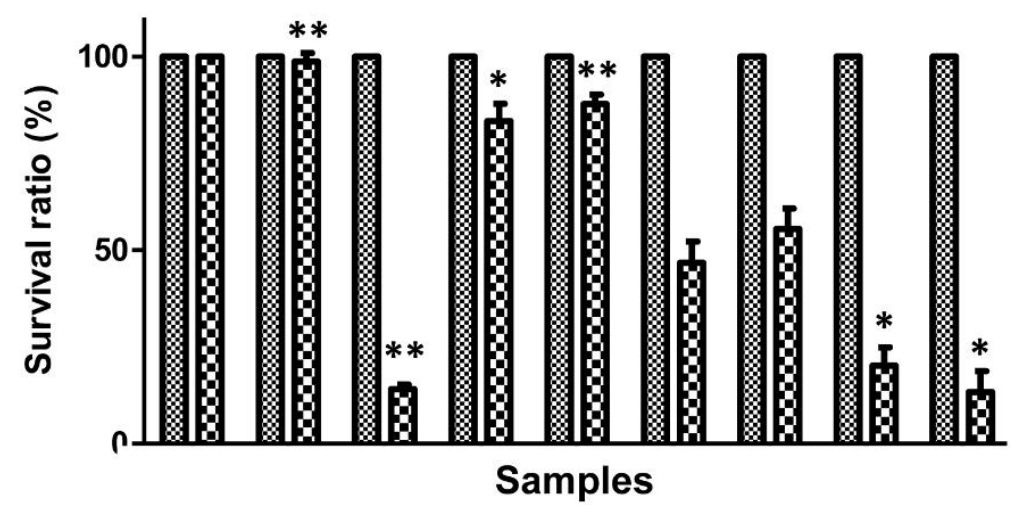

$\begin{array}{cccccccccc}\text { Month(M) } & 1 & 3 & 9 & 3 & 3 & 3 & 3 & 3 & 3 \\ \text { G.I. } & - & - & - & + & - & - & + & - & + \\ \text { Hypoxic } & - & - & - & - & - & + & - & + & + \\ \text { HFD } & - & - & - & - & + & - & + & + & +\end{array}$

Figure 6. Observation of the damaged $\mathrm{OHC}$ and IHC exposed to various conditions in the OC. The yellow arrow indicates OHC, and the white arrow indicates IHC in all images. (a-h) Microscope magnification $\times 20$, scale bar $=20 \mu \mathrm{M}$. (a) Young (4 weeks) (b) 3 months (c) 3 months (+GI) (d) 3 months (+HFD) (e) 3 months (+ hypoxia), (f) 3 months (+HFD, GI), (g) 3 months (+hypoxia, HFD), (h) 3 months (+hypoxia, HFD, GI), and (i) the survival ratio of OHC and IHC in the different groups. Significant values are shown for each month with ${ }^{*} \mathrm{p}<0.05,{ }^{* *} \mathrm{p}<0.005$.

We observed the appearance of hair cells to further examine the damage caused to hair cells from oxidative stress. The function and survival of auditory hair cells were determined by observing the appearance of stereocilia, based on literature [32]. Under the hypoxic condition, the OHC was partially broken (Figure 7). In addition, the damage to hair cells was severe when HFD and GI conditions were included through a triple reaction. It was observed that the stereocilia were almost entirely gone. This implies that auditory hair cells are damaged by oxidative stress under these conditions. 


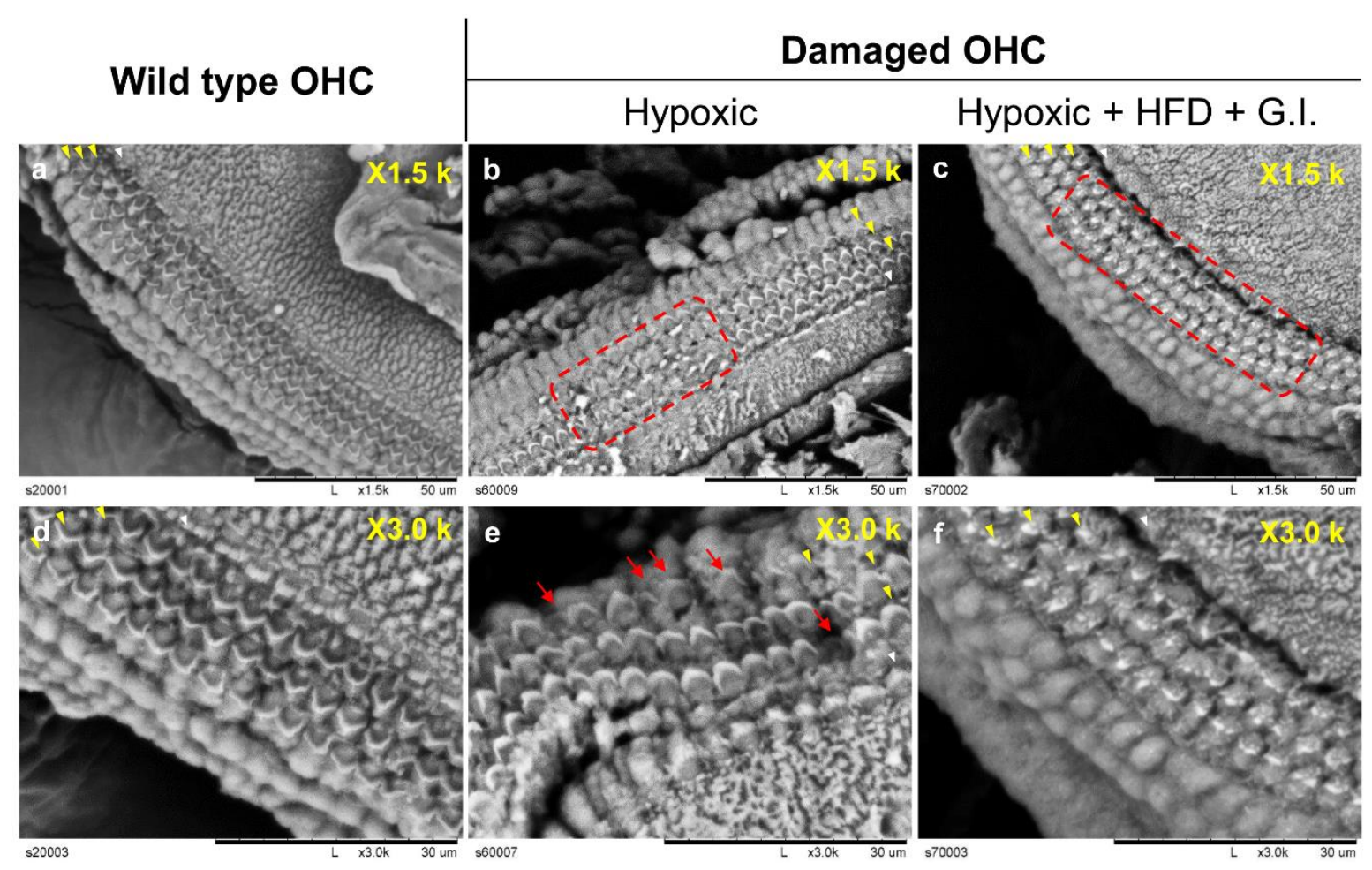

Figure 7. Images of survived OHC and IHC in the explant model by SEM. The yellow arrow indicates three OHC lines, and the white arrow indicates an IHC line. The red arrows indicate damaged hair cells. It was observed that the morphology of cilia disappeared on the line. The red dotted line indicates an extensive area of damaged hair cells. (a-c) microscope magnification $\times 1.5 \mathrm{k}$, scale bar $=50$ $\mu \mathrm{m}$, and (d-f) microscope magnification $\times 3.0 \mathrm{k}$, scale bar $=30 \mu \mathrm{m}$. (a) and (d) showed OHC extracted from a Young (4week). (b) and (e) show an OHC damaged by oxidative stress due to hypoxia. $\mathbf{c}$ and (f) show an $\mathrm{OHC}$ damaged by oxidative stress due to hypoxia, high fat diet, and galactose injection.

\subsection{Expression of age-related factors in cochlea}

We previously confirmed the occurrence of hearing loss due to the damage caused to hair cells by three kinds of environmental stresses. To demonstrate hearing loss and the damage caused to hair cells, we assessed whether the factors of aging are expressed in the auditory organ. We also sought to demonstrate the age-related hearing loss caused by environmental stresses in our animal model by identifying factors that are typically expressed in ARHL. The genes, ApoE [35-37] and EDN1 [38,39], are expressed under persistent oxidative stress conditions and have been reported to be associated with vascular aging (Figure 8). In addition, UCP2 is the most important gene and has been reported as a gene expressed in mitochondrial dysfunction [16]. $C D H 23$ is a gene expressed during typical ARHL [40,41]. Lastly, KCNQ4, Myo7a, Myo6, and SLC26A4 have been reported to be associated with the potassium channel and molecular physiological mechanisms of auditory organs [42-44]. All these genes are expressed during aging and are important markers that can be used to determine the cause of the expression of these genes [45].

The expression of all the selected genes had increased significantly under triple exposure conditions (Figure 8). Importantly, the expression of $U C P 2$, which is expressed during mitochondrial dysfunction due to oxidative stress, is significantly increased compared to that of other genes (Figure 8c). Furthermore, the expression of $\mathrm{CDH} 23$, which is the most expressed gene during aging of auditory organs, was found to be increased (Figure 8g). HFD is thought to induce the expression of $A p o E$ and $E D N 1$, which, although not very effective, were thought to aid hypoxic damage (Figure 8a). In the case of $A p o E$, the result of HFD and intermittent hypoxia alone showed similar expression level of RNA with treatment of the three conditions (Figure 8h). It was expected that HFD could induce hyperlipidaemia in the blood vessels of auditory organs. An imbalance in nutritional supply due to 
reduced blood flow has also been reported as a cause of hearing loss. Ion channel-related proteins in the auditory organs such as Slc24A4 (Figure 8b) and KCNQ4 (Figure 8d) were overexpressed. The expression of Myo7a and Myo6 increased significantly due to damage to the auditory organs (Figure $8 \mathrm{e}, \mathrm{f})$. Therefore, when stimulation by GI, intermittent hypoxic exposure, and HFD was not performed alone, the expression of aging factors was largely obtained.

a

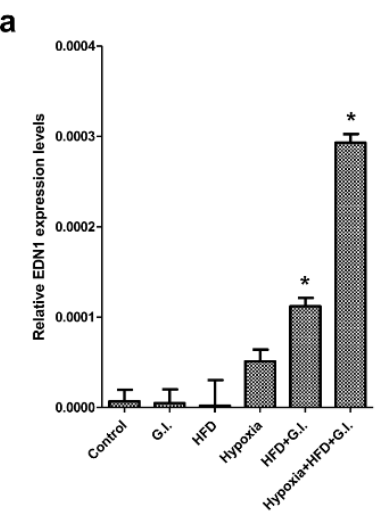

e

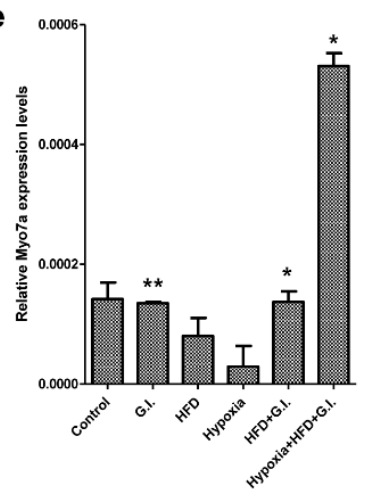

b

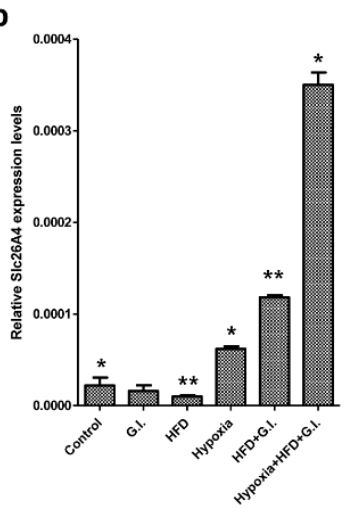

f 0.0006

c

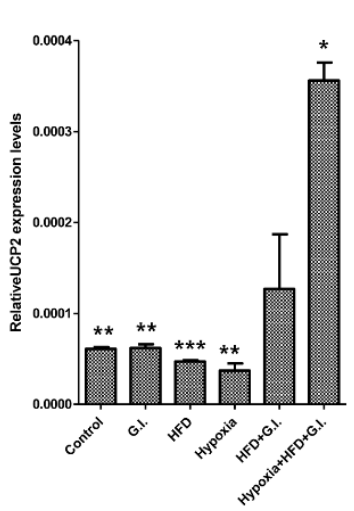

g

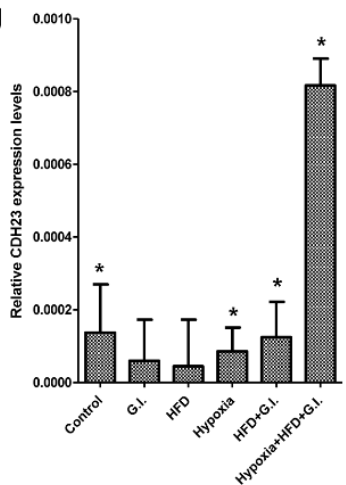

d

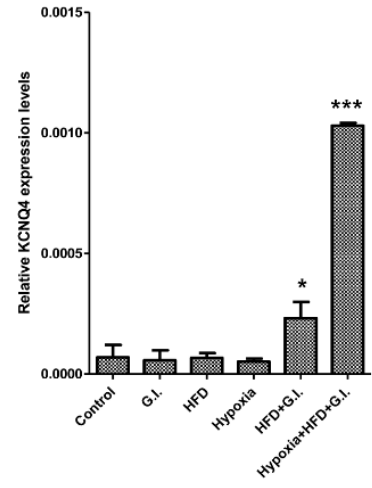

h

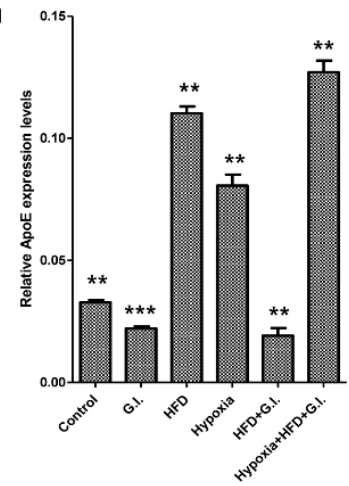

Figure 8: Expression of aging-related factors determined by real-time PCR. (a) EDN1, (b) Slc24A4, (c) UCP2, (d) KCNQ4, (e) Myo7a, (f) Myo6, (g) CDH23, and (h) ApoE. Samples from each group show aspects of control, GI, HFD, and hypoxia (single reaction) and HFD + hypoxia, hypoxia, HFD, and hypoxia, HFD, GI (complex reactions) from the left side. ${ }^{*} \mathrm{p}<0.05,{ }^{* *} \mathrm{p}<0.01,{ }^{* * *} \mathrm{p}<0.0001$.

\section{Discussion}

ARHL is a critical health condition affecting the aging population, and its onset varies based on the individual's lifestyle including eating and sleeping habits, noise exposure, and use of ototoxic drugs [4,7]. Studies have utilised specific genetically engineered mice or induced aging in animal models by drug injection, but these studies have been conducted without considering the changing conditions of the surrounding environment. This study describes the changes in hearing and histological phenotypes of hearing organs based on lifestyle. Herein, we showed that the expression of genes associated with aging-related deafness is largely induced over a short period of time, and these genes can be explored further in preventive or therapeutic research. In other words, since it is necessary to devise an animal model suitable for such studies, we proposed an animal model exposed to environmental stresses that can cause hearing loss in this study.

Hearing measurements are obtained is an easier manner using the C57BL/6 mice model than the other models. A recent study has shown that aging is caused by oxidative stress due to changes in lifestyle and has described strategies to prevent ARHL and to develop regenerative therapeutic substances [46]. This study aimed to reveal the phenotypes associated with hearing loss in a mouse model of hearing loss, without other diseases such as diabetes or vascular diseases. In our study, we observed that mice exposed to environmental stress for more than 3 months showed symptoms of diabetes or vascular disease; however, we did not investigate these observations further since they 
were beyond the scope of our study. In addition, persistent environmental stress is associated with poor quality of life, difficulty in communication, impaired activity in daily life, dementia, and cognitive dysfunction [11].

In this study, we used a total of three kinds of environmental stress stimuli. First, a hypoxia chamber was designed based on a hearing loss study on sleep habits such as OSAS [12]. When oxygen saturation decreases in the body during sleep, hearing decreases to $60 \mathrm{~dB}$ or more in patients over 60 years of age [11]. We demonstrated senile deafness caused by the variation in stratum corneum and wrinkles in the mouse model of oxidative stress. Change in lifestyle increases SOD in the serum and a reduction in atmospheric oxygen significantly influences aging. $\mathrm{CDH} 23$ was also expressed with triple exposure (Figure 8). The loss of auditory hair cells and the reduction in hearing ability were significantly reduced by the single stimuli, and when two or more stimuli were added, hearing loss was observed to occur over a short period of time. HFD significantly increases the content of fat in the body, affecting sugar metabolism and causing metabolic diseases and restriction of blood vessels. The results could be demonstrated by the expression of EDN1 and ApoE factors in cells within the hearing organ. HFD caused metabolic abnormalities in mice with hypoxia. Finally, $500 \mathrm{mg} / \mathrm{kg}$ galactose was injected to mice to induce aging through metabolic abnormalities in the body, and the effects of this administration were observed during HFD and hypoxia conditions. Thus, by determining the expression of UCP2 under all environmental stimuli, it was confirmed that mitochondrial dysfunction was caused by oxidative stress $[8,10]$. The study of mitochondria dysfunction has been observed to induce apoptosis in many studies, and we obtained similar results through histological analysis in this study $[15,16]$.

\section{Conclusions}

In summary, aging and physiological changes were induced by the three lifestyle conditions considered in this study. (Figure 9) Hypoxia has the greatest effect on hearing loss induction, while HFD and GI induce cell nutrient supply abnormalities caused by metabolic changes in the body to promote aging of cells (Table 2). Interestingly, HFD and GI (single exposure) led to no significant hearing loss. However, when hypoxia stimulation was added, the dual exposure showed intermediate effects, and the triple exposure showed the greatest effect. In addition, OHC loss occurs at high frequencies of sound when the blood vessels are abundant, and the exchange of substances in the bloodstream is poor.

This can cause ion channel abnormalities and genetic defects in the auditory organs [34,45]. We observed the age-related factors and phenotypes associated with ARHL in this study that caused natural short-term induction of ARHL through changes in the lifestyle due to oxidative stress. The animal model used and the results reported in this study can aid in the development of strategies for the prevention and treatment of ARHL. As the aging population increases in number, advances in medical technologies and research on hearing loss are warranted.

Table 2. Phenotypic changes caused by exposure to three oxidative stresses (hypoxia, HFD, GI).

\begin{tabular}{cccccccc}
\hline & \multicolumn{3}{c}{ Merged reaction } & \multicolumn{3}{c}{ Effect of a single factor } \\
\cline { 2 - 7 } & Triple & Dual & Single & Hypoxia & HFD & GI \\
\hline Phenotype & & & & & & & \\
Body weight & +++ & ++ & + & ++ & +++ & + \\
Skin thickness & +++ & ++ & + & ++ & +++ & + \\
Hair cell loss & +++ & ++ & + & +++ & ++ & + \\
Oxidative stress & +++ & ++ & + & +++ & ++ & + \\
Hearing loss & +++ & ++ & + & +++ & ++ & + \\
Age-related gene & +++ & ++ & + & +++ & ++ & + \\
expression & ++ & ++ & & & & & + \\
\hline
\end{tabular}




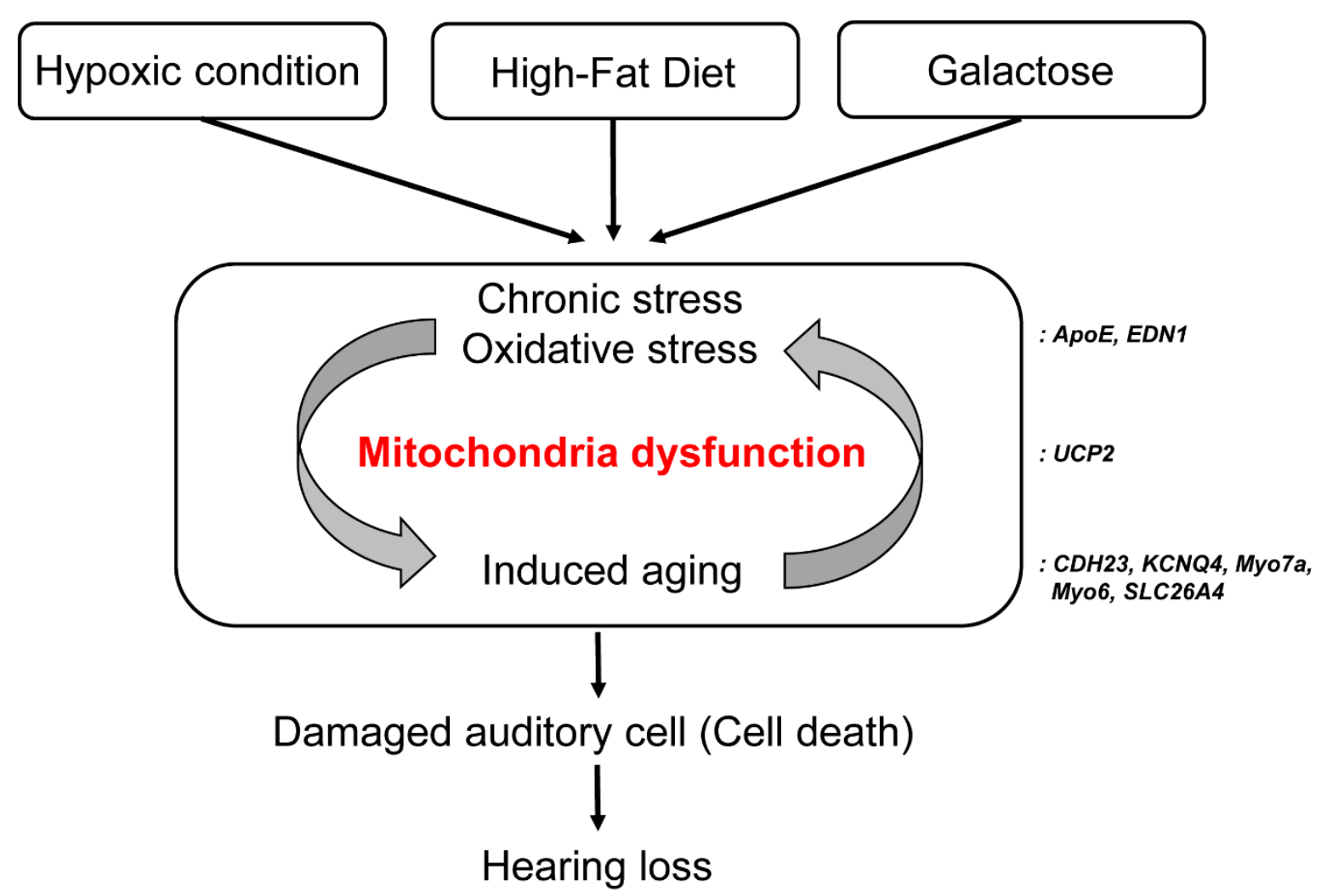

Figure 9. A schematic diagram of the aging mouse model exposed to three environmental oxidative stress factors. The three factors (hypoxia, HFD, and GI) cause mitochondrial dysfunction by inflicting oxidative stress on cells. Aged auditory hair cells accumulate due to irritation, followed by cell death and hearing loss.

Supplementary Materials: Supplementary materials can be found at www.mdpi.com/xxx/s1.

Author Contributions: Conceptualisation, D.J.P.; Methodology, D.J.P., S.H., J.S.C., S.H.L. and J.E.P.; Software, D.J.P. and S.H.; Data curation, S.H.; Formal analysis, J.S.C.; Resources, Y.J.S.; Writing - original draft, D.J.P.; Writing - review \& editing, S.H., J.S.C., S.H.L., J.E.P. and Y.J.S; Visualisation, D.J.P.; Project administration, Y.J.S; Funding Acquisition, D.J.P. and Y.J.S.

Funding: This research was supported by a grant from the Korea Health Technology R\&D Project through the Korea Health Industry Development Institute (KHIDI), funded by the Ministry of Health \& Welfare, Republic of Korea (grant number: HI19C1334).

Acknowledgments: We would like to thank Editage (www.editage.co.kr) for English language editing.

Conflicts of Interest: The authors declare no potential conflicts of interest with respect to the authorship and/or the publication of this article.

\section{Abbreviations}

ABR Auditory brainstem response

ARHL Age-related hearing loss

GI Galactose injection

H\&E Hematoxylin and eosin

HFD High-fat diet

IHC Inner hair cell

KHIDI Korea Health Industry Development Institute

NF Normal feed 


$\begin{array}{ll}\text { OC } & \text { Organ of Corti } \\ \text { OHC } & \text { Outer hair cell } \\ \text { OSAS } & \text { Obstructive sleep apnea syndrome } \\ \text { ROS } & \text { Reactive oxidative stress } \\ \text { RT } & \text { Room temperature }\end{array}$

\section{References}

1. Bowl, M.R.; Dawson, S.J. The mouse as a model for age-related hearing loss-a mini-review. Gerontology 2015, 61, 149-157, doi:10.1159/000368399.

2. Yamasoba, T.; Lin, F.R.; Someya, S.; Kashio, A.; Sakamoto, T.; Kondo, K. Current concepts in age-related hearing loss: Epidemiology and mechanistic pathways. Hear. Res. 2013, 303, 30-38, doi:10.1016/j.heares.2013.01.021.

3. Zelaya, C.E.; Lucas, J.W.; Hoffman, H.J. Self-reported hearing trouble in adults aged 18 and over: United states, 2014. Nchs data brief, no 214. National Center for Health Statistics: Hyattsville, MD, 2015.

4. Someya, S.; Xu, J.Z.; Kondo, K.; Ding, D.L.; Salvi, R.J.; Yamasoba, T.; Rabinovitch, P.S.; Weindruch, R.; Leeuwenburgh, C.; Tanokura, M., et al. Age-related hearing loss in c57bl/6j mice is mediated by bakdependent mitochondrial apoptosis. Proc. Natl. Acad. Sci. U. S. A. 2009, 106, 19432-19437, doi:10.1073/pnas.0908786106.

5. Johnson, K.R.; Tian, C.; Gagnon, L.H.; Jiang, H.; Ding, D.; Salvi, R. Effects of cdh23 single nucleotide substitutions on age-related hearing loss in c57bl/6 and 129s1/sv mice and comparisons with congenic strains. Sci. Rep. 2017, 7, 44450, doi:10.1038/srep44450.

6. White, K.; Kim, M.J.; Han, C.; Park, H.J.; Ding, D.; Boyd, K.; Walker, L.; Linser, P.; Meneses, Z.; Slade, C., et al. Loss of idh2 accelerates age-related hearing loss in male mice. Sci. Rep. 2018, 8, 5039, doi:10.1038/s41598018-23436-w.

7. Ren, H.M.; Ren, J.; Liu, W. Recognition and control of the progression of age-related hearing loss. Rejuvenation Res. 2013, 16, 475-486, doi:10.1089/rej.2013.1435.

8. Liu, X.Z.; Yan, D. Ageing and hearing loss. J. Pathol. 2007, 211, 188-197, doi:10.1002/path.2102.

9. Kim, S.H.; Yeo, S.G. Presbycusis. Hanyang Med. Rev. 2015, 35, 78-83, doi:10.7599/hmr.2015.35.2.78.

10. Hildesheimer, M.; Rubinstein, M.; Nuttal, A.L.; Lawrence, M. Influence of blood viscosity on cochlear action potentials and oxygenation. Hear. Res. 1982, 8, 187-198, doi:10.1016/0378-5955(82)90074-0.

11. Nash, S.D.; Cruickshanks, K.J.; Klein, R.; Klein, B.E.; Nieto, F.J.; Huang, G.H.; Pankow, J.S.; Tweed, T.S. The prevalence of hearing impairment and associated risk factors: The beaver dam offspring study. Arch. Otolaryngol. Head Neck Surg. 2011, 137, 432-439, doi:10.1001/archoto.2011.15.

12. Seo, Y.J.; Park, S.Y.; Chung, H.J.; Kim, C.H.; Lee, J.G.; Kim, S.H.; Cho, H.J. Lowest oxyhemoglobin saturation may be an independent factor influencing auditory function in severe obstructive sleep apnea. Journal of Clinical Sleep Medicine 2016, 12, 653-658, doi:10.5664/jcsm.5786.

13. Hao, S.; Wang, L.; Zhao, K.; Zhu, X.; Ye, F. Rs1894720 polymorphism in miat increased susceptibility to age-related hearing loss by modulating the activation of mir-29b/sirt1/pgc-1alpha signaling. J. Cell. Biochem. 2019, 120, 4975-4986, doi:10.1002/jcb.27773.

14. Seidman, M.D.; Ahmad, N.; Joshi, D.; Seidman, J.; Thawani, S.; Quirk, W.S. Age-related hearing loss and its association with reactive oxygen species and mitochondrial DNA damage. Acta Otolaryngol. Suppl. 2004, Suppl 552, 16-24, doi:10.1080/03655230410017823. 
15. Chen, H.; Tang, J. The role of mitochondria in age-related hearing loss. Biogerontology 2014, 15, 13-19, doi:10.1007/s10522-013-9475-y.

16. Fujimoto, C.; Yamasoba, T. Oxidative stresses and mitochondrial dysfunction in age-related hearing loss. Oxid. Med. Cell. Longev. 2014, 2014, 582849, doi:10.1155/2014/582849.

17. Fujita, T.; Yamashita, D.; Uehara, N.; Inokuchi, G.; Hasegawa, S.; Otsuki, N.; Nibu, K. A high-fat diet delays age-related hearing loss progression in c57bl/6j mice. PLoS One 2015, 10, e0117547, doi:10.1371/journal.pone.0117547.

18. Kume, S.; Uzu, T.; Horiike, K.; Chin-Kanasaki, M.; Isshiki, K.; Araki, S.; Sugimoto, T.; Haneda, M.; Kashiwagi, A.; Koya, D. Calorie restriction enhances cell adaptation to hypoxia through sirt1-dependent mitochondrial autophagy in mouse aged kidney. J. Clin. Invest. 2010, 120, 1043-1055, doi:10.1172/Jci41376.

19. Mitchell, P.; Gopinath, B.; McMahon, C.M.; Rochtchina, E.; Wang, J.J.; Boyages, S.C.; Leeder, S.R. Relationship of type 2 diabetes to the prevalence, incidence and progression of age-related hearing loss. Diabet. Med. 2009, 26, 483-488, doi:10.1111/j.1464-5491.2009.02710.x.

20. Guo, B.; Guo, Q.; Wang, Z.; Shao, J.B.; Liu, K.; Du, Z.D.; Gong, S.S. D-galactose-induced oxidative stress and mitochondrial dysfunction in the cochlear basilar membrane: An in vitro aging model. Biogerontology 2020, 21, 311-323, doi:10.1007/s10522-020-09859-x.

21. Ho, S.C.; Liu, J.H.; Wu, R.Y. Establishment of the mimetic aging effect in mice caused by d-galactose. Biogerontology 2003, 4, 15-18, doi:10.1023/a:1022417102206.

22. Liao, C.H.; Chen, B.H.; Chiang, H.S.; Chen, C.W.; Chen, M.F.; Ke, C.C.; Wang, Y.Y.; Lin, W.N.; Wang, C.C.; Lin, Y.H. Optimizing a male reproductive aging mouse model by d-galactose injection. Int. J. Mol. Sci. 2016, 17, 98, doi:10.3390/ijms17010098.

23. Parameshwaran, K.; Irwin, M.H.; Steliou, K.; Pinkert, C.A. D-galactose effectiveness in modeling aging and therapeutic antioxidant treatment in mice. Rejuvenation Res. 2010, 13, 729-735, doi:10.1089/rej.2010.1020.

24. Reeves, P.G.; Nielsen, F.H.; Fahey, G.C., Jr. Ain-93 purified diets for laboratory rodents: Final report of the american institute of nutrition ad hoc writing committee on the reformulation of the ain-76a rodent diet. J. Nutr. 1993, 123, 1939-1951, doi:10.1093/jn/123.11.1939.

25. Seo, Y.J.; Ju, H.M.; Lee, S.H.; Kwak, S.H.; Kang, M.J.; Yoon, J.H.; Kim, C.H.; Cho, H.J. Damage of inner ear sensory hair cells via mitochondrial loss in a murine model of sleep apnea with chronic intermittent hypoxia. Sleep 2017, 40, zsx106, doi:10.1093/sleep/zsx106.

26. Ju, H.M.; Lee, S.H.; Choi, J.S.; Seo, Y.J. A simple model for inducing optimal increase of sdf-1 with aminoglycoside ototoxicity. Biomed. Res. Int. 2017, 2017, 4630241, doi:10.1155/2017/4630241.

27. Chatuphonprasert, W.; Lao-Ong, T.; Jarukamjorn, K. Improvement of superoxide dismutase and catalase in streptozotocin-nicotinamide-induced type 2-diabetes in mice by berberine and glibenclamide. Pharm. Biol. 2013, 52, 419-427, doi:10.3109/13880209.2013.839714.

28. Kim, Y.Y.; Nam, H.; Jung, H.; Kim, B.; Suh, J.G. Over-expression of myosin7a in cochlear hair cells of circling mice. Lab. Anim. Res. 2017, 33, 1-7, doi:10.5625/lar.2017.33.1.1.

29. Riva, C.; Donadieu, E.; Magnan, J.; Lavieille, J.P. Age-related hearing loss in cd/1 mice is associated to ros formation and hif target proteins up-regulation in the cochlea. Exp. Gerontol. 2007, 42, 327-336, doi:10.1016/j.exger.2006.10.014.

30. Potter, P.K.; Bowl, M.R.; Jeyarajan, P.; Wisby, L.; Blease, A.; Goldsworthy, M.E.; Simon, M.M.; Greenaway, S.; Michel, V.; Barnard, A., et al. Novel gene function revealed by mouse mutagenesis screens for models of age-related disease. Nat. Commun. 2016, 7, 12444, doi:10.1038/ncomms12444. 
31. Alameda, J.P.; Ramirez, A.; Garcia-Fernandez, R.A.; Navarro, M.; Page, A.; Segovia, J.C.; Sanchez, R.; Suarez-Cabrera, C.; Paramio, J.M.; Bravo, A., et al. Premature aging and cancer development in transgenic mice lacking functional cyld. Aging (Albany NY) 2019, 11, 127-159, doi:10.18632/aging.101732.

32. Bourguignon, L.Y.; Wong, G.; Xia, W.; Man, M.Q.; Holleran, W.M.; Elias, P.M. Selective matrix (hyaluronan) interaction with cd44 and rhogtpase signaling promotes keratinocyte functions and overcomes age-related epidermal dysfunction. J. Dermatol. Sci. 2013, 72, 32-44, doi:10.1016/j.jdermsci.2013.05.003.

33. Orioli, D.; Dellambra, E. Epigenetic regulation of skin cells in natural aging and premature aging diseases. Cells 2018, 7, 268, doi:10.3390/cells7120268.

34. Raynor, L.A.; Pankow, J.S.; Miller, M.B.; Huang, G.H.; Dalton, D.; Klein, R.; Klein, B.E.; Cruickshanks, K.J. Familial aggregation of age-related hearing loss in an epidemiological study of older adults. Am. J. Audiol. 2009, 18, 114-118, doi:10.1044/1059-0889(2009/08-0035).

35. Guo, Y.; Zhang, C.; Du, X.; Nair, U.; Yoo, T.J. Morphological and functional alterations of the cochlea in apolipoprotein e gene deficient mice. Hear. Res. 2005, 208, 54-67, doi:10.1016/j.heares.2005.05.010.

36. Kim, Y.Y.; Chao, J.R.; Kim, C.; Kim, B.; Nguyen, P.T.T.; Jung, H.; Chang, J.; Lee, J.H.; Suh, J.G. Hearing loss through apoptosis of the spiral ganglion neurons in apolipoprotein e knockout mice fed with a western diet. Biochem. Biophys. Res. Commun. 2020, 523, 692-698, doi:10.1016/j.bbrc.2019.12.100.

37. Kurniawan, C.; Westendorp, R.G.; de Craen, A.J.; Gussekloo, J.; de Laat, J.; van Exel, E. Gene dose of apolipoprotein e and age-related hearing loss. Neurobiol. Aging 2012, 33, 2230 e2237-2230 e2212, doi:10.1016/j.neurobiolaging.2012.04.001.

38. Bondurand, N.; Dufour, S.; Pingault, V. News from the endothelin-3/ednrb signaling pathway: Role during enteric nervous system development and involvement in neural crest-associated disorders. Dev. Biol. 2018, 444 Suppl 1, S156-S169, doi:10.1016/j.ydbio.2018.08.014.

39. Uchida, Y.; Sugiura, S.; Nakashima, T.; Ando, F.; Shimokata, H. Endothelin-1 gene polymorphism and hearing impairment in elderly japanese. Laryngoscope 2009, 119, 938-943, doi:10.1002/lary.20181.

40. Bouzid, A.; Smeti, I.; Chakroun, A.; Loukil, S.; Gibriel, A.A.; Grati, M.; Ghorbel, A.; Masmoudi, S. Cdh23 methylation status and presbycusis risk in elderly women. Front. Aging Neurosci. 2018, 10, 241, doi:10.3389/fnagi.2018.00241.

41. Usami, S.I.; Nishio, S.Y.; Moteki, H.; Miyagawa, M.; Yoshimura, H. Cochlear implantation from the perspective of genetic background. Anat. Rec. (Hoboken) 2020, 303, 563-593, doi:10.1002/ar.24360.

42. Alimardani, M.; Hosseini, S.M.; Khaniani, M.S.; Haghi, M.R.; Eslahi, A.; Farjami, M.; Chezgi, J.; Derakhshan, S.M.; Mojarrad, M. Targeted mutation analysis of the slc26a4, myo6, pjvk and cdh23 genes in iranian patients with ar nonsyndromic hearing loss. Fetal Pediatr. Pathol. 2019, 38, 93-102, doi:10.1080/15513815.2018.1547336.

43. Holme, R.H.; Steel, K.P. Stereocilia defects in waltzer (cdh23), shaker1 (myo7a) and double waltzer/shaker1 mutant mice. Hear. Res. 2002, 169, 13-23, doi:10.1016/s0378-5955(02)00334-9.

44. Park, H.J.; Shaukat, S.; Liu, X.Z.; Hahn, S.H.; Naz, S.; Ghosh, M.; Kim, H.N.; Moon, S.K.; Abe, S.; Tukamoto, K., et al. Origins and frequencies of slc26a4 (pds) mutations in east and south asians: Global implications for the epidemiology of deafness. J. Med. Genet. 2003, 40, 242-248, doi:10.1136/jmg.40.4.242.

45. Muller, U.; Barr-Gillespie, P.G. New treatment options for hearing loss. Nat. Rev. Drug Discov. 2015, 14, 346365, doi:10.1038/nrd4533.

46. Wang, J.; Puel, J.L. Presbycusis: An update on cochlear mechanisms and therapies. J. Clin. Med. 2020, 9, 218, doi:10.3390/jcm9010218. 